MODELING, IDENTIFICATION AND CONTROL, 1990, vOL. 11, NO. 1, 55-72

doi: $10.4173 /$ mic.1990.1.5

\title{
A Schur method for designing $L Q$-optimal systems with prescribed eigenvalues
}

\author{
DAVID DI RUSCIO $\dagger$ and JENS G. BALCHEN $\dagger$
}

Keywords: Linear optimal regulator, pole placement, multivariable control systems, control system design, computer-aided design, robustness.

\begin{abstract}
In this paper a new algorithm for solving the $L Q$-optimal pole placement problem is presented. The method studied is a variant of the classical eigenvector approach and instead uses a set of Schur vectors, thereby gaining substantial numerical advantages. An important task in this method is the $L Q$-optimal pole placement problem for a second order (sub) system. The paper presents a detailed analytical solution to this problem. This part is not only important for solving the general $n$-dimensional problem but also provides an understanding of the behaviour of an optimal system: The paper shows that in some cases it is an infinite number; in others a finite number; and in still others, non state weighting matrices $Q$ that give the system a set of prescribed eigenvalues. Equations are presented that uniquely determine these state weight matrices as a function of the new prescribed eigenvalues. From this result we have been able to derive the maximum possible imaginary part of the eigenvalues in an $L Q$-optimal system, irrespective of how the state weight matrix is chosen.
\end{abstract}

\section{Introduction}

In this paper a new method for the determination of state weighting matrices, $Q$, is presented such that the closed loop system gets a set of prescribed eigenvalues and the feedback is a solution from the matrix Riccati equation. The problem of designing feedback gains to optimally place the poles can be divided into two classes.

The first class is to design the $L Q$-regulator by which all poles of the closed loop system are located in a specified region. This problem is discussed, among others, by Anderson and Moore (1969), Kawasaki and Shimemura (1983, 1988), Heger and Frank (1984), Shieh et al. (1986), Shieh et al. (1988).

Anderson and Moore (1969) placed the poles of the $L Q$-optimal closed loop system to the left of a vertical line on the negative real axis in the complex plane. Shieh et al. (1986) placed the eigenvalues in a vertical strip on the negative real axis. Kawasaki and Shimemura $(1983,1988)$ developed a recursive method for optimally placing the eigenvalues of the closed loop system in an open hyperbola of the complex plane, while Shieh et al. (1988) optimally placed the poles in an open sector of the complex plane. The hyperbola by Kawasaki et al. is an approximation of the sector by Shieh $e$ al .

The second class (to be studied in this paper) is to design the $L Q$-regulator by which the closed loop system gets prescribed eigenvalues. This problem is discussed, among others, by Graupe (1972), Solheim (1972), Bar-Ness (1978), Juang and Lee (1984), Amin (1985), Medanic et al. (1988), Saif (1989), and Tharp (1989).

Received 1 December 1989.

$\dagger$ Division of Engineering Cybernetics, Norwegian Institute of Technology, N-7034, Trondheim, Norway. 
Solheim (1972) presented a method based on an eigenvector approach. This method performs well for a system with real distinct eigenvalues if the system is well behaved and therefore can be diagonalized from a numerical point of view. If the system has complex eigenvalues which have to be moved by Solheim's method, this is of little interest since the method neglects important solutions. If the system cannot be diagonalized (a necessary condition is that the system has multiple eigenvalues) the old algorithm cannot be used directly. The Jordan canonical form was proposed as a solution to this problem. But this is only of theoretical interest because the Jordan canonical form is very difficult to determine numerically, see for example Gulub (1983) pp. 196-198. It also turns out that the algorithm solves an unnecessary problem, namely the solution of full-order non-linear Riccati equations for modifying one real or two complex eigenvalues in each step.

Graupe (1972) and Bar-Ness (1978) presented methods based on the sensitivity of the state weight matrix with respect to the eigenvalues. Juang and Lee (1984) used the modal control approach by Varga (1981) to determine a feedback gain matrix such that the closed loop system gets specified eigenvalues. Then an inverse control approach (Kalman 1964) is used to determine a possible corresponding state weight matrix. The method is based on Theorem 1 and Corollary 1 of Juang and Lee (1984). Amin and Hassan (1985) shows that neither the theorem nor the corollary can be correct.

Amin (1985) considered the same problem as Solheim (1972) and developed a method based on the results of Anderson and Moore (1969), and the mirror-image property by Moliniari (1977). In this method it is only possible to change the real parts of the eigenvalues while the imaginary parts are preserved. This could lead to a requirement of the solution of a linear Lyapunov equation rather than a non-linear Riccati equation. Medanic et al. (1988) used the negative definite solutions of the Riccati equation to modify the state weight matrix and thereby position the real parts of the eigenvalues while the imaginary parts are preserved. Tharp (1989) used the method by Medanic et al. (1988) on linear discrete systems. Saif(1989) used the result by Solheim (1972), Medanic et al. (1988) and Amin (1985). In this method it is possible to change the real and the imaginary parts of the eigenvalues, however, there are unnecessary restrictions on the position of the new imaginary part of the eigenvalues. Saif used the results by Solheim to move complex eigenvalues. The method is therefore conservative, unnecessary restrictions are therefore put on the performance matrices. Nothing new is added in the paper by Saif.

The method presented here is an extension of the work by Solheim (1972). The method by Solheim (1972), and the other methods mentioned above, are not general with respect to the position of the new eigenvalues. One reason is that unnecessary restrictions are taken on the matrices in the performance criteria. The relation between these matrices and the closed loop eigenvalues are of interest. In this paper we will extend the relations given by Solheim, and present the general solution to this problem. From this result we have been able to derive the maximum possible imaginary part of the eigenvalues in an $L Q$-optimal system, irrespective of how the state weight matrix $Q$ is chosen. This result completes the optimal pole placement problem, because it gives us the unique region where the poles can be located. In our method we are able to move two eigenvalues, real or complex conjugate, to new real or complex conjugate locations at each step, and only a Riccati equation of an order of not more than two has to be solved. The method may also be used to shift poles in an already optimal system to more desirable locations. 
The Schur method presented here has considerable numerical advantages compared to the diagonalization approach. The algorithm is firstly, completely general to the system structure and secondly, completely general to the structure of the eigenvalue spectrum. The reduction to real Schur form is an intermediate step in computing eigenvectors (using the double Francis $Q R$ algorithm) so the Schur approach must, by definition, be faster. Furthermore, for a second order system the algorithm is fundamental, that means that all solutions (state weight matrices) $Q$ are derived such that the closed loop spectrum gets specified eigenvalues.

The real Schur decomposition in control system design is used by Laub (1979) and Varga (1981). Laub (1979) used the real Schur decomposition to solve the algebraic Riccati equation, while Varga (1981) used the real Schur decomposition for solving the modal control problem, for example, designing a feedback gain without constraints such that the closed loop system gets prescribed eigenvalues.

The rest of the paper is organized as follows. Section 2 provides some definitions and theorems from linear algebra prior to obtaining the method. Section 3 provides some basics from control theory and the problem definitions are stated. Section 4 shows that the problem can be solved using a block triangulation approach. We show that the $n$-dimensional problem can be solved by decoupling a sub-system. Section 5 solves the problem for a second order general (sub) system. This is part of the $n$-dimensional problem. Equations are derived that determine all state weighting matrices $Q$ (if there are any) that give the $L Q$-optimal system some prescribed eigenvalues. The results are presented in Algorithm 5.1. Section 6 states a general algorithm, Algorithm 6.1, for the $n$-dimensional problem. Numerous examples are given in Section 7 and some concluding remarks are made in Section 8.

\section{Linear algebra review}

Before presenting the result some definitions and theorems are prepared for obtaining the method. Proofs are given in Golub (1983) Ch. 7.

Definition 1. $U \in \mathbf{R}^{n \times n}$ is orthogonal if $U^{T}=U^{-1}$.

Definition 2. $U \in \mathbf{C}^{n \times n}$ is unitary if $U^{H}=U^{-1}$.

Definition 3. $F \in \mathbf{R}^{2 n \times 2 n}$ is Hamiltonian if $L^{-1} F^{T} L=-F$, where $L$ is given below

$$
L=\left[\begin{array}{cc}
0 & I \\
-I & 0
\end{array}\right] \in \mathbf{R}^{2 n \times 2 n}
$$

$I$ denotes the $n \times n$ dimensional identity matrix. Note that $L^{T}=L^{-1}=-L$.

Theorem 1. Schur Decomposition (SD). Let $A \in \mathbf{R}^{n \times n}$. Then there exists a unitary similarity transformation $U$ such that $U^{H} A U=T$ is upper triangular with the eigenvalues on the diagonal of $T$. Furthermore, $U$ can be chosen such that the eigenvalues $\lambda_{i}$ appear in any desired order along the diagonal.

Theorem 2. Real Schur Decomposition (RSD). Let $A \in \mathbf{R}^{n \times n}$. Then there exists an orthogonal similarity transformation $U$ such that $U^{T} A U=T$ is quasi upper triangular. The diagonal blocks in $T$ consist of $1 \times 1$ blocks corresponding to real eigenvalues and $2 \times 2$ blocks corresponding to complex conjugate eigenvalues. Furthermore, $U$ can be chosen such that the $2 \times 2$ and $1 \times 1$ diagonal blocks appear in any desired order. 


\section{Basic definition}

Consider a time invariant dynamic system

$$
\underline{\dot{x}}=A \underline{x}+B \underline{u}
$$

and the quadratic objective functional

$$
J=\frac{1}{2} \int_{0}^{\infty}\left(\underline{x}^{T} Q \underline{x}+\underline{u}^{T} P \underline{u}\right) \mathrm{d} t
$$

where $\underline{x}$ is an $n$-dimensional state vector, $\underline{u}$ is an $r$-dimensional control input vector, $A$ and $B$ are constant matrices of appropriate dimensions and $Q$ and $P$ are $n \times n$ symmetrical and $r \times r$ positive definite symmetrical matrices respectively. The problem is to design a controller matrix $G$ of dimension $r \times n$ so that the closed loop system (4) gets a set of prescribed eigenvalues and such that the performance criterion (3) is minimized

$$
\underline{\dot{x}}=(A+B G) \underline{x}
$$

The optimal control that minimizes criterion (3) is given by

$$
\underline{u}=G \underline{x} \quad G=-P^{-1} B^{T} R
$$

where $R$ is a solution to the algebraic Riccati equation

$$
A^{T} R+R A-R H R+Q=0, \quad H=B P^{-1} B^{T}
$$

The Hamiltonian matrix is derived from optimal control theory by augmenting the co-states to the state space model (2). The Hamiltonian is

$$
F=\left[\begin{array}{cc}
A & -H \\
-Q & -A^{T}
\end{array}\right] \in \mathbf{R}^{2 n \times 2 n}
$$

$F$ has $2 n$ eigenvalues, ( $n$ stable and $n$ unstable) located symmetrically about the imaginary axis. The stable eigenvalues are identical to the eigenvalues of the closed loop system matrix $(A+B G)=(A-H R)$. This can be seen from the following similarity transformation

$$
\left[\begin{array}{ll}
I & 0 \\
R & I
\end{array}\right]^{-1}\left[\begin{array}{cc}
A & -H \\
-Q & -A^{T}
\end{array}\right]\left[\begin{array}{ll}
I & 0 \\
R & I
\end{array}\right]=\left[\begin{array}{cc}
A-H R & -H \\
0 & -(A-H R)^{T}
\end{array}\right]
$$

This means that the closed loop eigenvalue spectrum can be derived from $F$ without solving the algebraic Riccati equation. We can now transform the eigenvalue problem by similarity transformation. Using the following orthogonal similarity transformation we get

$$
\tilde{F}=\bar{U} F \bar{U}^{T}=\left[\begin{array}{cc}
U^{T} & 0 \\
0 & U^{T}
\end{array}\right]\left[\begin{array}{cc}
A & -H \\
-Q & -A^{T}
\end{array}\right]\left[\begin{array}{cc}
U & 0 \\
0 & U
\end{array}\right]=\left[\begin{array}{cc}
T & -\tilde{H} \\
-\tilde{Q} & -T^{T}
\end{array}\right]
$$

where $T, \tilde{Q}$ and $\tilde{H}$ are given by

$$
T=U^{T} A U \quad \tilde{Q}=U^{T} Q U \quad \tilde{H}=U^{T} H U
$$

This means that the $L Q$-problem in (2) and (3) is transformed to a new state space by the transformation $\underline{x}=U \underline{z}$ where $U$ is an orthogonal matrix and $\underline{z}$ is the new state vector. The new system matrix $T$ which is similar to $A$ with respect to the eigenvalues, the new state weight matrix $\tilde{Q}$ is given in (10). The control input matrix for this system is $U^{T} B$. 
Choosing $U$ as an orthogonal matrix that transforms $A$ by similarity transformation to an upper triangular Schur form $T$, then the eigenvalue dependence upon the state weighting matrix $Q$ can be derived from $\tilde{F}$ in $(9)$. This will be shown in the next section.

\section{Solution by block triangulization}

The eigenvalue dependence as a function of $Q$ in an $L Q$-optimal system can be derived from the following Hamiltonian matrix (canonical system)

$$
\tilde{F}=\left[\begin{array}{cc}
T & -\tilde{H} \\
-\tilde{Q} & -T^{T}
\end{array}\right]
$$

the system matrix $T$, now in real Schur form (RSD) and $\tilde{H}$ can be written

$$
T=\left[\begin{array}{cc}
T_{11} & T_{12} \\
0 & T_{22}
\end{array}\right] \in \mathbf{R}^{n \times n} \quad \tilde{H}=\left[\begin{array}{cc}
H_{11} & H_{12} \\
H_{12}^{T} & H_{22}
\end{array}\right] \in \mathbf{R}^{n \times n}
$$

$T_{11}$ is an $n-2 \times n-2$ dimensional RSD matrix with $1 \times 1$ or $2 \times 2$ diagonal blocks. The $1 \times 1$ blocks correspond to real eigenvalues and the $2 \times 2$ blocks to complex conjugate eigenvalues. The matrix $T_{22}$ is a $2 \times 2$ dimensional RSD block of either two $1 \times 1$ diagonal blocks or a general real $2 \times 2$ block. We note that if $T_{22}$ consists of $1 \times 1$ blocks then the system modus number $n$ in $T$ is decoupled. Then $\lambda_{n}$ can be moved directly by Solheim's algorithm (Solheim 1972). This is only a special case of our algorithm.

Block $T_{22}$ is decoupled from the other elements in $T$. The eigenvalues of the general $2 \times 2$ block $T_{22}$ can be moved while the other eigenvalues are unchanged by the following state weight matrix $Q$. Proof is given below.

$$
\tilde{Q}=\left[\begin{array}{cc}
0 & 0 \\
0 & Q_{22}
\end{array}\right]
$$

where $Q_{22}$ is a $2 \times 2$ symmetric matrix (not necessary non-negative). We will now derive expressions for the eigenvalues of (11). The eigenvalues of $\tilde{F}$ are given by the characteristic polynomial of (11)

$$
\operatorname{det}(s I-\tilde{F})=\operatorname{det}\left[\begin{array}{cccc}
s I-T_{11} & -T_{12} & H_{11} & H_{12} \\
0 & s I-T_{22} & H_{12}^{T} & H_{22} \\
0 & 0 & s I+T_{11}^{T} & 0 \\
0 & Q_{22} & T_{12}^{T} & s I+T_{22}^{T}
\end{array}\right]
$$

If we develop the determinant (14) from the first column and the third row we get the expression

$$
\operatorname{det}(s I-\widetilde{F})=\operatorname{det}\left(s I-T_{11}\right) \operatorname{det}\left(s I+T_{11}^{T}\right) \operatorname{det}\left(s I-F_{22}\right)
$$

where $F_{22}$ is a $4 \times 4$ Hamiltonian matrix corresponding to the decoupled sub-system $T_{22}$ given by

$$
F_{22}=\left[\begin{array}{cc}
T_{22} & -H_{22} \\
-Q_{22} & -T_{22}^{T}
\end{array}\right]
$$


Using the Schur formula (given in the Appendix) on the third part on the right-hand side in equation (15) we get an alternative expression

$$
\operatorname{det}(s I-\widetilde{F})=\operatorname{det}\left[\left(s I-T_{11}\right)\left(s I+T_{11}^{T}\right)\left(s I-T_{22}\right)\left(s I+T_{22}^{T}-Q_{22}\left(s I-T_{22}\right)^{-1} H_{22}\right)\right]
$$

Before we continue we will mention a special case. Assume the scalar case where $T_{22}=\lambda_{n}, Q_{22}=q_{n n}$ and $H_{22}=h_{n n}$. Then from (15) or (17) we get

$$
q_{n n}=\frac{s_{n}^{2}-\lambda_{n}^{2}}{h_{n n}}, \quad h_{n n} \neq 0
$$

This was the main result in Solheim's (1972) algorithm.

The problem is now reduced to determining the eigenvalue dependence upon the weight matrix $Q_{22}$ in the general $4 \times 4$ dimensional Hamiltonian matrix $F_{22}$. In the next section we will derive analytic expressions for the state weight matrix $Q_{22}$ such that the decoupled sub-system in position $T_{22}$ gets prescribed eigenvalues. These results are very important for studying the behaviour of an optimal system and do not seem to be known, or mentioned in the literature. Another important conclusion from this result is that when $\widetilde{Q}$ has the form (13) and $T$ and $\tilde{H}$ have the form in (12) then we do not have to solve a Riccati equation whose order is more than the dimension of the decoupled system (16). We also recall that there exists an orthogonal $U$ such that the diagonal blocks in $T$ appear in any desired order. This means that any diagonal block $T_{i i}$ in $T_{11}$ whose eigenvalues are to be moved can be placed in position $T_{22}$ with an orthogonal similarity transformation (Stewart 1976).

\section{The second order problem}

In this section we will derive analytic expressions for the problem of determining state weight matrices $Q$ such that a second order optimal system gets two new prescribed eigenvalues.

The Hamilton matrix that determines the closed loop eigenvalues is given by

$$
F=\left[\begin{array}{cc}
T & -H \\
-Q & -T^{T}
\end{array}\right]
$$

where $T, Q$ and $H$ are given by

$$
T=\left[\begin{array}{ll}
t_{11} & t_{12} \\
t_{21} & t_{22}
\end{array}\right] \quad H=\left[\begin{array}{ll}
h_{11} & h_{12} \\
h_{12} & h_{22}
\end{array}\right] \quad Q=\left[\begin{array}{ll}
q_{11} & q_{12} \\
q_{12} & q_{22}
\end{array}\right]
$$

The characteristic polynomial of the Hamiltonian matrix $F$ is given by

$$
L(s)=s^{4}-p_{1} s^{2}+p_{2}=0
$$

The polynomial coefficients in terms of the state weight matrix elements are given by

$$
\begin{aligned}
& p_{1}=b_{1} q_{11}+b_{2} q_{22}+d_{1} q_{12}+d_{2} \\
& p_{2}=a_{0} q_{11} q_{22}+a_{1} q_{11}+a_{2} q_{22}-a_{0} q_{12}^{2}+k_{1} q_{12}+k_{2}
\end{aligned}
$$

where the coefficients are only dependent on the matrices $T$ and $H$. The coefficients are given in the Appendix. The eigenvalues can either consist of real or complex conjugate 
pairs. If we prescribe the eigenvalues of the Hamiltonian, the polynomial coefficients in (21) for these two cases would be

Case 1. Real eigenvalues $\lambda_{1}$ and $\lambda_{2}$

$$
\begin{aligned}
& p_{1}=\lambda_{1}^{2}+\lambda_{2}^{2} \\
& p_{2}=\lambda_{1}^{2} \lambda_{2}^{2} .
\end{aligned}
$$

Case 2. Complex conjugate eigenvalues $\lambda_{1}=\alpha+j \beta$ and $\lambda_{2}=\alpha-j \bar{\beta}$

$$
\begin{aligned}
& p_{1}=2\left(\alpha^{2}-\beta^{2}\right) \\
& p_{2}=\left(\alpha^{2}+\beta^{2}\right)^{2}
\end{aligned}
$$

We now have the problem of solving two equations with three unknowns. We choose $q_{12}$ as a free parameter and get the following equations from (22) and (23) that determine $q_{11}$ and $q_{22}$

$$
\begin{aligned}
a_{0} q_{11} q_{22}+a_{1} q_{11}+a_{2} q_{22}+a_{3} & =0 \\
b_{1} q_{11}+b_{2} q_{22}+b_{3} & =0
\end{aligned}
$$

where $a_{3}$ and $b_{3}$ are functions of $q_{12}$ and are given by

$$
\begin{aligned}
& a_{3}=-a_{0} q_{12}^{2}+k_{1} q_{12}+k_{2}-p_{2} \\
& b_{3}=d_{1} q_{12}+d_{2}-p_{1}
\end{aligned}
$$

We will first discuss the case when $a_{0}=\operatorname{det} H \neq 0$. Given $q_{12}$ as a free parameter we can solve equations (28) and (29) for $q_{11}$ and $q_{22}$. These equations could be written

$$
\begin{aligned}
& a_{0} b_{1} q_{11}^{2}+\left(a_{0} b_{3}-a_{1} b_{2}+a_{2} b_{1}\right) q_{11}+a_{2} b_{3}-a_{3} b_{2}=0 \\
& a_{0} b_{2} q_{22}^{2}+\left(a_{0} b_{3}+a_{1} b_{2}-a_{2} b_{1}\right) q_{22}+a_{1} b_{3}-a_{3} b_{1}=0
\end{aligned}
$$

We are only interested in the real solutions. These equations have real solutions if and only if (surprisingly we get only one inequality from equations (32) and (33))

$$
\begin{aligned}
J_{I}\left(q_{12}\right) & =\left(a_{0} b_{3}-a_{1} b_{2}+a_{2} b_{1}\right)^{2}-4 a_{0} b_{1}\left(a_{2} b_{3}-a_{3} b_{2}\right) \\
& =\left(a_{0} b_{3}+a_{1} b_{2}-a_{2} b_{1}\right)^{2}-4 a_{0} b_{2}\left(a_{1} b_{3}-a_{3} b_{1}\right) \\
& \geqslant 0
\end{aligned}
$$

We recall that the coefficients $a_{3}$ and $b_{3}$ are functions of $q_{12}$ while the others are constant given by $t_{i j}$ and $h_{i j}$. If we develop (34) in terms of $q_{12}$ we determine the fundamental inequality that constrains $q_{12}$. The inequality is given below.

$$
J_{I}\left(q_{12}\right)=c_{1} q_{12}^{2}+c_{2} q_{12}+c_{3} \geqslant 0
$$

where the coefficient $c_{1}$ is constant and only dependent on the matrix elements in H. $c_{1}$ is easily determined by the equality below

$$
c_{1}=-4(\operatorname{det} H)^{3}
$$

When det $H>0$ then $J_{I}\left(q_{12}\right)$ has a maximum value. This means that there must be a finite solution interval of (35) if there is any at all. If $\operatorname{det} H<0$ then $J_{I}\left(q_{12}\right)$ have a minimum value and there would be an infinite interval if there is any at all. But when $H=H^{T}<0$ then the solution $R$ of the Riccati equation is not necessarily positive 
definite. The coefficients $c_{2}$ and $c_{3}$ are dependent on the coefficients of the new prescribed polynomial $p_{1}$ and $p_{2}$. Where the relation between the new prescribed eigenvalues and $p_{1}$ and $p_{2}$ is given in Case 1 and Case 2 above. We can now write coefficients $c_{2}$ and $c_{3}$ in terms of these coefficients

$$
\begin{aligned}
& c_{2}=g_{1} p_{1}+g_{2} \\
& c_{3}=f_{1} p_{1}^{2}+f_{2} p_{1}+f_{3}+f_{4} p_{2}
\end{aligned}
$$

The coefficients $g_{i}$ and $f_{i}$ are again only dependent on $T$ and $H$. See the Appendix for details.

If $a_{0}=\operatorname{det} H=0$, then the second order system of polynomial equations (28) and (29) will degenerate to a second order system of linear equations. The solution is given by

$$
\left[\begin{array}{l}
q_{11} \\
q_{22}
\end{array}\right]=\frac{1}{a_{1} b_{2}-a_{2} b_{1}}\left[\begin{array}{rr}
b_{2} & -a_{2} \\
-b_{2} & a_{1}
\end{array}\right]\left[\begin{array}{l}
-k_{1} q_{12}-k_{2}+p_{2} \\
-d_{1} q_{12}-d_{2}+p_{1}
\end{array}\right]
$$

Equation (39) can be written

$$
\begin{aligned}
& q_{11}=v_{1} q_{12}+v_{2} \\
& q_{22}=w_{1} q_{12}+w_{2}
\end{aligned}
$$

where the coefficients $v_{i}$ and $w_{i}$ are given in the Appendix. When $a_{1} b_{2}-a_{2} b_{1}=0$ then there is no solution to equation (39). Physically this means that the linear system model is uncontrollable (see comments in Section 6). We see from equations (40) and (41) that there will be an infinite number of solutions. However, if we require that $Q=Q^{T} \geqslant 0$ then we will get a finite number of solutions or none. In this case we will get an inequality $\left(q_{11} q_{22}-q_{12}^{2}=c_{4} q_{12}^{2}+c_{5} q_{12}+c_{6} \geqslant 0\right)$ for $q_{12}$ with the same form as (35). It is indeed clear that it could be necessary with a negative $Q$, for example when the new eigenvalues are specified right to the eigenvalues of the original system in the complex plane. Note that the eigenvalues can be specified all over the left complex plane, including the imaginary axis.

We can now summarize the above results in a general algorithm for a second order linear time invariant system. This algorithm is again part of the overall $n$-dimensional system in Section 6.

\section{Algorithm 5.1}

Step 1. Given a second order system described by $T$ and $H . T$ can be a single system matrix and $H=B P^{-1} B^{T}$, or $T$ could be a decoupled $2 \times 2$ block from a $n$-dimensional system and $H$ the corresponding $2 \times 2$ diagonal block.

Step 2. Compute coefficients $a_{i}, b_{i}, c_{1}, d_{i}, g_{i}$ and $f_{i}$ as functions of the elements of the matrices $T$ and $H$. The coefficients are given in the Appendix.

Step 3. Specify two eigenvalues. Compute the coefficients $p_{1}$ and $p_{2}$.

Step 4. If $a_{0}=\operatorname{det} H=0$, then compute solutions from equations (40) and (41) and terminate, else jump to Step 5.

Step 5. Compute coefficients $c_{2}$ and $c_{3}$ that depend on the new prescribed eigenvalues from

$$
\begin{aligned}
& c_{2}=g_{1} p_{1}+g_{2} \\
& c_{3}=f_{1} p_{1}^{2}+f_{2} p_{1}+f_{3}+f_{4} p_{2}
\end{aligned}
$$


Step 6. Check if the proposed $q_{12}$ satisfies the inequality

$$
J_{I}\left(q_{12}\right)=c_{1} q_{12}^{2}+c_{2} q_{12}+c_{3} \geqslant 0
$$

If this inequality is not satisfied, determine a $q_{12}$ that does. If it is not possible to satisfy this inequality it means that it is not possible to determine $L Q$-optimal system with these pole locations. Recall that the real parts of the eigenvalues can be located all along the real axis, but that the imaginary value is physically limited. See equation (49) that uniquely determines the maximum possible imaginary value, no matter how $Q$ are chosen.

Step 7. Compute coefficient $b_{3}$ as a function of $q_{12}$

$$
b_{3}=d_{1} q_{12}+d_{2}-p_{1}
$$

Step 8. Compute the diagonal elements in $Q$. For each choice of $q_{12}$ we get two generally different sets of weighting matrices. The two sets are marked with super index $\alpha$ and $\beta$. It is assumed that $a_{0} \neq 0$ and that the system is controllable, then $b_{1} \neq 0$ and $b_{2} \neq 0$

$$
\begin{aligned}
& q_{11}^{\alpha}=\frac{-a_{0} b_{3}+\left(a_{1} b_{2}-a_{2} b_{1}\right)-\sqrt{ }\left(J_{I}\left(q_{12}\right)\right)}{2 a_{0} b_{1}} \\
& q_{22}^{\alpha}=\frac{-a_{0} b_{3}-\left(a_{1} b_{2}-a_{2} b_{1}\right)+\sqrt{ }\left(J_{I}\left(q_{12}\right)\right)}{2 a_{0} b_{2}} \\
& q_{11}^{\beta}=\frac{-a_{0} b_{3}+\left(a_{1} b_{2}-a_{2} b_{1}\right)+\sqrt{ }\left(J_{I}\left(q_{12}\right)\right)}{2 a_{0} b_{1}} \\
& q_{22}^{\beta}=\frac{-a_{0} b_{3}-\left(a_{1} b_{2}-a_{2} b_{1}\right)-\sqrt{ }\left(J_{I}\left(q_{12}\right)\right)}{2 a_{0} b_{2}}
\end{aligned}
$$

We see that the following relations hold

$$
q_{22}^{\alpha}=\frac{-b_{3}-h_{11} q_{11}^{\alpha}}{h_{22}} \quad q_{22}^{\beta}=\frac{-b_{3}-h_{11} q_{11}^{\beta}}{h_{22}} \quad \frac{q_{11}^{\alpha}-q_{11}^{\beta}}{q_{22}^{\beta}-q_{22}^{\alpha}}=\frac{h_{22}}{h_{11}}
$$

The final result is given by

$$
Q^{\alpha}=\left[\begin{array}{ll}
q_{11}^{\alpha} & q_{12} \\
q_{12} & q_{22}^{\alpha}
\end{array}\right] \quad Q^{\beta}=\left[\begin{array}{ll}
q_{11}^{\beta} & q_{12} \\
q_{12} & q_{22}^{\beta}
\end{array}\right]
$$

The following comments can be made about this algorithm.

When $a_{0}=\operatorname{det} H \neq 0$ then we see from (44) that the relation between $q_{11}$ and $q_{22}$ is linear. The relations between $q_{i i}, i=1,2$ and $q_{12}$ can be found from (42) and (43). These relations are elliptic when the solution of the inequality in Step 6 is finite. Then sets $\alpha$ and $\beta$ are two parts of this ellipse. The relations are generally given by

$$
m_{1 i} q_{i i}^{2}+m_{2 i} q_{i i} q_{12}+m_{3 i} q_{12}^{2}+m_{4 i} q_{i i}+m_{5 i} q_{12}+m_{6 i}=0, \quad i=1,2
$$

where $m_{j i}, j=1,6$ is easily found from $a_{i}, b_{i}, c_{i}, d_{i}$ and $p_{i}$. When $a_{0}=\operatorname{det} H=0$, then the relation between $q_{i i}, i=1,2$ and $q_{12}$ is linear. This is seen from equations (40) and (41). When $a_{0}=\operatorname{det} H<0$, then a sign is changed and the elliptic relation above is changed to a hyperbolic relation between $q_{i i}, i=1,2$ and $q_{12}$. This is (of course) of theoretical interest because $H=H^{T} \geqslant 0$ when $P=P^{T}>0$. See the comments in Section 6. 
This method gives the answer to the question about whether it is possible with a given control weight matrix $P$ to determine a state weight matrix $Q$ so that the closed loop system obtained a set of prescribed eigenvalues. This method does not just provide a yes/no answer but gives us all such matrices. When $\boldsymbol{H}=\boldsymbol{H}^{\boldsymbol{T}}<0$, then we will either have an infinite number of solutions or none, but in this case we cannot be sure that the solution of the Riccati equation is positive.

This method can be used to determine all state weight matrices $Q$ (if there are none) that correspond to a set of prescribed eigenvalues. It is known that the behaviour of a system under feedback not only is dependent on the eigenvalues but also on the eigenvectors. As a result of this method we have the freedom to choose the corresponding eigenvectors such that some performance criteria are optimized. One such could be the quadratic performance criterion

$$
\min _{Q} J=\operatorname{tr}(R), \quad E\left(x(0) x^{T}(0)\right)=I
$$

Another such criterion could be the Frobenius norm for the closed loop system matrix

$$
\min _{Q} J_{F}=(A+B G)_{F}=\left(\sum_{i=1}^{n} \sum_{j=1}^{n}\left(\bar{a}_{i j}\right)^{2}\right)^{1 / 2}
$$

Where $\bar{a}_{i j}$ indicates element $i j$ in $A+B G$. The relation between this norm and the robustness in the sense of an additive uncertainty $d A$ in the system matrix $A$ is given in Dickman (1987). It can be shown that the robustness is maximized when the Frobenius norm for the closed-loop system is minimized. This means that we can allow larger perturbations $d A$ when the Frobenius norm is minimized. It can also be shown that the minimization up to $\sqrt{ }\left(\Sigma_{i=1}^{n}\left(\lambda_{i}\right)^{2}\right)$ is possible. This could happen if there are enough degrees of freedom in the controller to make $A+B G$ symmetrical. The structured singular value $\mu$ (Doyle et al. 1982) could also be used. A criterion which will be investigated is one which is directly connected to the eigenvalue sensitivity. Because of this algorithm it is now up to the designer to choose a suitable performance criterion.

Here we will present the equation that uniquely determines the maximum possible imaginary part of the eigenvalues in an $L Q$-system, irrespective of how $Q$ are chosen. The derivation is restricted to a second order system, because the general solution is a topic of a further paper. The derivation is also restricted to the case where $a_{0}=\operatorname{det} H \neq 0$, because there is generally no upper limit on the imaginary value when $a_{0}=\operatorname{det} H=0$.

If we develop the inequality (35) in terms of $b=\beta^{2}$ and optimize with respect to $\beta$, then after some algebra we get

$$
\beta_{\max }^{2}=b_{\max }=\frac{\left[\left(h_{12} a_{22}+h_{11} a_{21}\right)-\left(h_{22} a_{12}+h_{12} a_{11}\right)\right]^{2}}{4 \operatorname{det} H} \geqslant 0
$$

This means that the imaginary value $\beta$ always is bounded by

$$
0 \leqslant|\beta| \leqslant \sqrt{ }\left(b_{\max }\right)
$$

We see that the maximum imaginary value only is dependent on the matrices $A$ and $H=B P^{-1} B^{T}$, and not on the real parts of the closed loop eigenvalues. Note that $\beta_{\max }$ goes to infinity when $a_{0}=\operatorname{det} H$ goes to zero. This means that there is no upper limit on $\beta$ when $a_{0}=\operatorname{det} H=0$. But if we restrict the state weight matrix to $Q \geqslant 0$ then there will be a maximum value. In this case $\beta$ will be dependent on the real value $\alpha$. This result 
completes the pole placement problem, because it gives us the unique region where the eigenvalues can be located.

\section{The $n$-dimensional problem}

In this section we present the results from Sections 4 and 5 in a general algorithm for the determination of state weight matrix $Q$ such that an optimal system gets a set of prescribed eigenvalues.

\section{Algorithm 6.1}

Step 1. Initialize $Q=Q_{i}$ and $R=R_{i}, i=0$. Compute $H=B P^{-1} B^{T}$.

Step 2. Compute the closed loop system matrix $A_{i}=A-H R$. (Equivalent to $A+B G$.) If system order $0<n \leqslant 2$, jump to Step 6 .

Step 3. Compute Real Schur Decomposition $U, T=U^{T} A_{i} U$.

Step 4. Specify one or two eigenvalues $s_{1}$ and $s_{2}$ to be shifted and the corresponding diagonal block $T_{j j}$. Reorder the RSD decomposition with an orthogonal $U_{D}$ such that the block $T_{j j}$ is placed in position $T_{22}$ (lower right corner in $T$ ). Update the orthogonal transformation $U=U U_{D}$.

Step 5. Compute $\tilde{H}=U^{T} H U$. Only block $H_{22}$ in position in the lower right corner is needed.

Step 6. Use Algorithm 5.1 to compute $Q_{22}$ from the decoupled sub-system given by $T_{22}$ and $H_{22}$. for $R_{22}$

Step 7. Solve the algebraic Riccati equation (which is of a maximum order of two)

$$
T_{22}^{T} R_{22}+R_{22} T_{22}-R_{22} H_{22} R_{22}+Q_{22}=0
$$

Step 8. Back-transform solutions from

$$
Q_{i}=U \tilde{Q} U^{T} \quad R_{i}=U \tilde{R} U^{T}
$$

where

$$
\tilde{Q}=\left[\begin{array}{cc}
0 & 0 \\
0 & Q_{22}
\end{array}\right] \quad \tilde{R}=\left[\begin{array}{cc}
0 & 0 \\
0 & R_{22}
\end{array}\right]
$$

Step 9. Update $R$ and the state weight matrix $Q$

$$
Q=Q+Q_{i} \quad R=R+R_{i}
$$

Step 10. If all eigenvalues are shifted, then compute feedback matrix

$$
G=-P^{-1} B^{T} R,
$$

or update $A=A_{i}$ and $i=i+1$ and go to Step 2 . The following comments can be made about this algorithm.

In Step 2 in the algorithm we suggest omitting Steps 3,4 and 5 when the order of the system is two or less. As we have shown in Section 5, the problem can be solved directly for a second order system.

In Step 3 the RSD decomposition must be computed. We know that the computation of RSD is an intermediate step in computing the diagonal or block- 
diagonal decomposition. The reordering of block $T_{j j}$ to position $T_{22}$ is trivial(see Golub (1983) pp. 240-242 and Stewart (1976) for details). From this we see that the RSD with the reordering of block $T_{j j}$ is faster than block diagonalizing $T$, for example. Another important fact is that only to take the RSD decomposition will eliminate the problems and hazards there are to diagonalizing a defective matrix with multiple or nearmultiple eigenvalues.

In Steps 6 and 7 we recommend working with a second order sub-system because the eigenvalues of the block can be moved directly. During this step we get all possible weight matrices $Q$, and therefore the corresponding eigenvectors can be optimized. See Section 5 for details.

We see only one Riccati equation of a maximum order of two is to be solved. This is an easy task and can be done analytically. In our algorithm we do it numerically, but it would be interesting to check if computational work and numerical precision could be saved. We recall that in the scalar case where $T_{22}=\lambda_{n}, Q_{22}=q_{n n}$ and $H_{22}=h_{n n}$, then $q_{n n}$ can be computed from (18) and only a scalar Riccati equation has to be solved.

When the control input weight matrix $P=P^{T}>0$ then $H=B P^{-1} B^{T} \geqslant 0$. When the system is controllable then it can be shown that the diagonal elements in $H$ are always non-negative, and that the block $H_{22}$ is non-negative. When the mode $z_{n}$ is uncontrollable, then the $n$th row of $U^{T} B$ contains only zero elements, and the $n$th row and $n$th column in $H$ contains only zero elements.

\section{Numerical examples}

\subsection{Example 1}

Consider the system

$$
A=\left[\begin{array}{rr}
-2 & 0 \\
1 & -1
\end{array}\right] \quad B=\left[\begin{array}{ll}
1 & 0 \\
0 & 1
\end{array}\right] \quad P=\left[\begin{array}{ll}
1 & 0 \\
0 & 5
\end{array}\right] \quad H=\left[\begin{array}{ll}
1 & 0 \\
0 & 0-2
\end{array}\right]
$$

The system eigenvalues are

$$
\lambda_{1}(A)=-2, \quad \lambda_{2}(A)=-1
$$

The eigenvalues of the optimal closed loop system are specified as

$$
s_{1}(A+B G)=-8, \quad s_{2}(A+B G)=-5
$$

We use Algorithm 5.1 directly and find that $q_{12}$ must satisfy the inequality

$$
J_{I}\left(q_{12}\right)=-0.032 q_{12}^{2}+0 \cdot 32 q_{12}+96 \cdot 64 \geqslant 0
$$

The inequality is satisfied if and only if

$$
-50 \cdot 18 \leqslant q_{12} \leqslant 60 \cdot 18
$$

We can now plot $q_{11}, q_{22}$ and the measure of the performance index $J=\operatorname{tr}(R)$ as a function of $q_{12}$ when the closed loop system has fixed eigenvalues. This is illustrated in Fig. (1). We have also plotted the Frobenius norm $(A+B G)_{F}$.

For this example we have an elliptical relation between $q_{i i}, i=1,2$ and $q_{12}$. If we use $J=\operatorname{tr}(R)$ as a measure of optimality we get one solution, where the value is $J_{\min }=\operatorname{tr}(R)=24 \cdot 52$.

$$
Q^{\beta}=\left[\begin{array}{cc}
62 \cdot 18 & 16 \cdot 03 \\
16 \cdot 03 & 109 \cdot 10
\end{array}\right] \quad R=\left[\begin{array}{cc}
6 \cdot 370 & 2 \cdot 629 \\
2 \cdot 629 & 18 \cdot 150
\end{array}\right] \quad G=\left[\begin{array}{cc}
-6 \cdot 370 & -2 \cdot 629 \\
-0.526 & -3.630
\end{array}\right]
$$



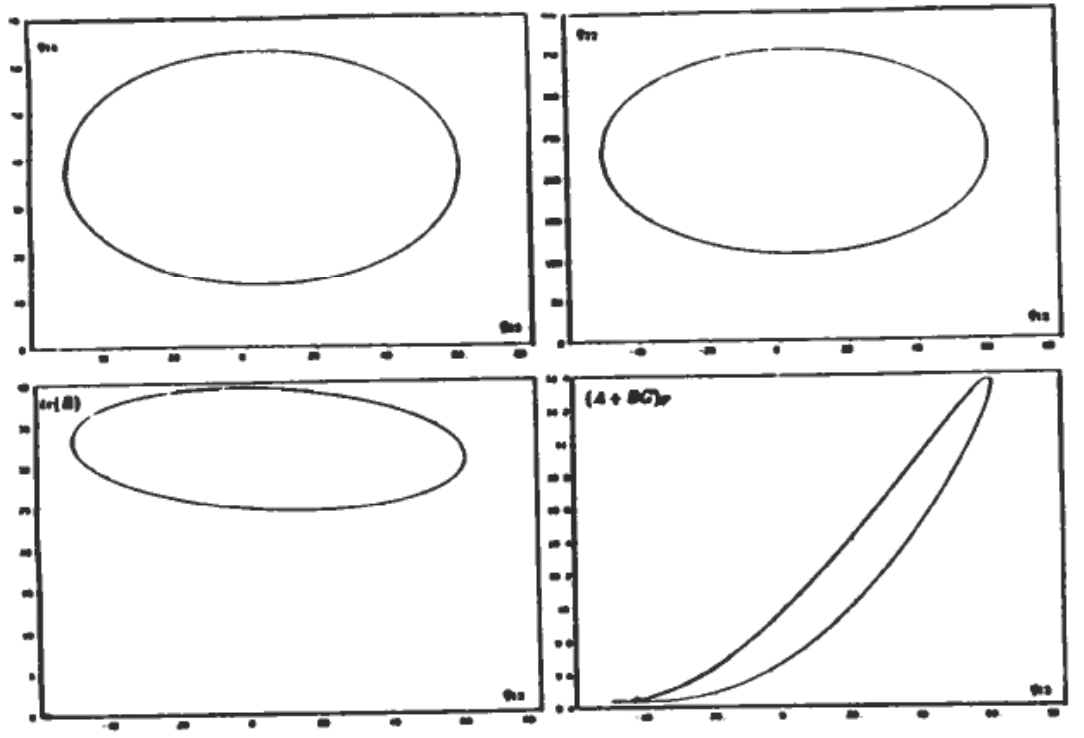

Figure 1. Plot of $q_{11}, q_{22}, J=\operatorname{tr}(R)$ and $(A+B G)_{F}$ as a function of $q_{12}$.

We also note that we have two diagonal solutions to the problem, namely

$$
Q^{\alpha}=\left[\begin{array}{cc}
13 \cdot 42 & 0 \\
0 & 352 \cdot 88
\end{array}\right] \quad Q^{\beta}=\left[\begin{array}{cc}
62 \cdot 58 & 0 \\
0 & 107 \cdot 12
\end{array}\right]
$$

The corresponding values of the performance criteria are $\operatorname{tr}\left(R^{\alpha}\right)=39.46$ and $\operatorname{tr}\left(R^{\beta}\right)=24 \cdot 77$.

\subsection{Example 2}

Consider the system

$$
A=\left[\begin{array}{rr}
-2 & 1 \\
-1 & -2
\end{array}\right] \quad B=\left[\begin{array}{ll}
2 & 1 \\
2 & 3
\end{array}\right] \quad P=\left[\begin{array}{ll}
1 & 0 \\
0 & 5
\end{array}\right] \quad H=\left[\begin{array}{ll}
4 \cdot 2 & 4 \cdot 6 \\
4 \cdot 6 & 5 \cdot 8
\end{array}\right]
$$

The system eigenvalues are

$$
\lambda_{1}(A)=-2+i, \quad \lambda_{2}(A)=-2-i
$$

The eigenvalues of the optimal closed loop system are specified as

$$
s_{1}(A+B G)=-8, \quad s_{2}(A+B G)=-5
$$

We use Algorithm 5.1 directly and find that $q_{12}$ must satisfy the inequality

$$
J_{I}\left(q_{12}\right)=-131 \cdot 1 q_{12}^{2}-21264 \cdot 4 q_{12}-234155 \cdot 5 \geqslant 0
$$

The inequality is satisfied if and only if

$$
-150 \cdot 35 \leqslant q_{12} \leqslant-11 \cdot 88
$$

We can now plot $q_{11}, q_{22}$ and the measure of the performance index $J=\operatorname{tr}(R)$ as a function of $q_{12}$ when the closed loop system has fixed eigenvalues. This is illustrated in Fig. 2. We have also plotted the Frobenius norm $(A+B G)_{F}$. 

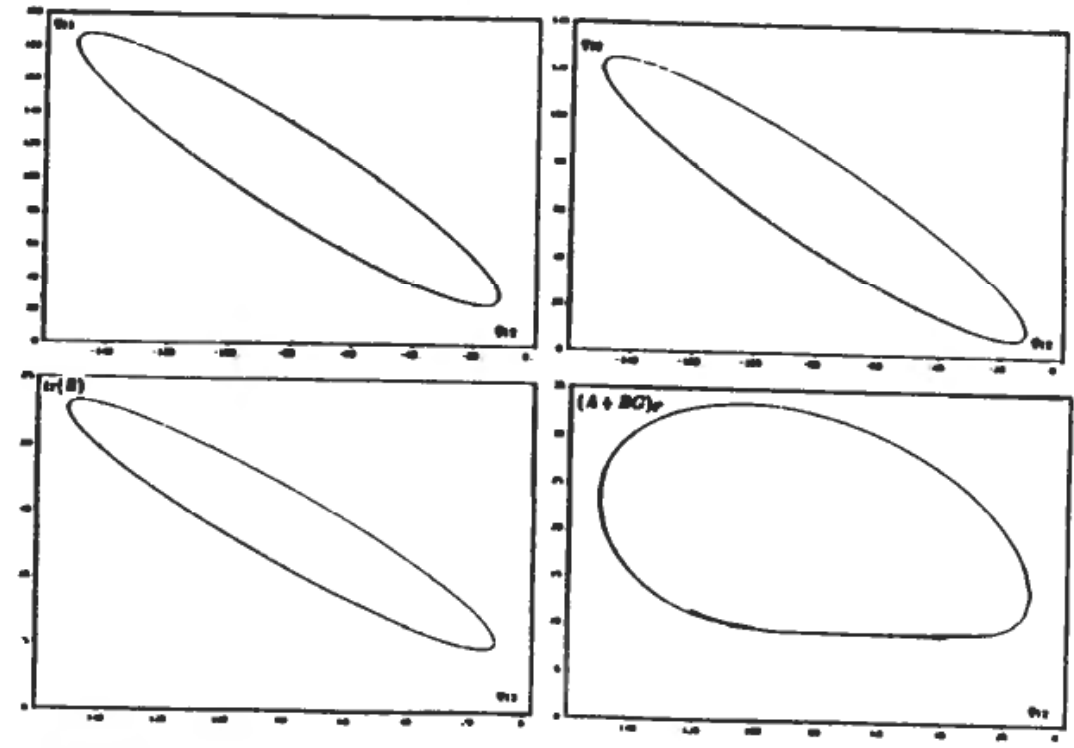

Figure 2. Plot of $q_{11}, q_{22}, J=\operatorname{tr}(R)$ and $(A+B G)_{F}$ as a function of $q_{12}$.

For this example we have an elliptical relation between $q_{i i}, i=1,2$ and $q_{12}$. If we use $J=\operatorname{tr}(R)$ as a measure of optimality we get one solution, where the value is $J_{\min }=\operatorname{tr}(R)=4 \cdot 81$.

$$
Q^{\beta}=\left[\begin{array}{rr}
42 \cdot 83 & -15 \cdot 13 \\
-15.13 & 7 \cdot 30
\end{array}\right] \quad R=\left[\begin{array}{rr}
4 \cdot 056 & -1 \cdot 349 \\
-1.349 & 0.755
\end{array}\right] \quad G=\left[\begin{array}{rr}
-5.413 & 1 \cdot 188 \\
-0.002 & -0.183
\end{array}\right]
$$

There are no diagonal solutions for this example.

\subsection{Example 3}

Consider the system

$$
A=\left[\begin{array}{ll}
0 & 1 \\
0 & 0
\end{array}\right] \quad B=\left[\begin{array}{l}
0 \\
1
\end{array}\right] \quad P=1 \quad H=\left[\begin{array}{ll}
0 & 0 \\
0 & 1
\end{array}\right]
$$

The system eigenvalues are

$$
\lambda_{1}(A)=0, \quad \lambda_{2}(A)=0
$$

The eigenvalues of the optimal closed loop system are specified as

$$
s_{1}(A+B G)=-1, \quad s_{2}(A+B G)=-1
$$

For this system $\operatorname{det}(H)=0$. We would first solve this problem by working with a scalar subsystem (Algorithm 6.1). From (17) we get $q_{22}=1$. The solution of the scalar Riccati equation is $r=1$.

$$
Q_{1}=\tilde{Q}=\left[\begin{array}{ll}
0 & 0 \\
0 & 1
\end{array}\right] \quad R_{1}=\tilde{R}=\left[\begin{array}{ll}
0 & 0 \\
0 & 1
\end{array}\right] \quad G_{1}=\left[\begin{array}{ll}
0 & -1
\end{array}\right] \quad A_{1}=\left[\begin{array}{rr}
0 & 1 \\
0 & -1
\end{array}\right]
$$

$A_{1}$ is in real Schur form. To move the other eigenvalue we must reorder the Schur form. 
The reordered Schur decomposition of $A_{1}$ gives

$$
T=\left[\begin{array}{cc}
-1 & 1 \\
0 & 0
\end{array}\right] \quad U=\left[\begin{array}{cc}
\frac{1}{\sqrt{ } 2} & \frac{1}{\sqrt{ } 2} \\
-\frac{1}{\sqrt{ } 2} & \frac{1}{\sqrt{ } 2}
\end{array}\right] \quad \tilde{H}=U^{T} H U=\left[\begin{array}{rr}
\frac{1}{2} & -\frac{1}{2} \\
-\frac{1}{2} & \frac{1}{2}
\end{array}\right]
$$

The same procedure as above gives $q_{22}=2, r=2$ and

$$
Q_{2}=U \tilde{Q} U^{T}=\left[\begin{array}{ll}
1 & 1 \\
1 & 1
\end{array}\right] \quad R_{2}=U \tilde{R} U^{T}=\left[\begin{array}{ll}
1 & 1 \\
1 & 1
\end{array}\right] \quad G_{2}=\left[\begin{array}{ll}
-1 & -1
\end{array}\right]
$$

The final results are given by

$$
Q=Q_{1}+Q_{2}=\left[\begin{array}{ll}
1 & 1 \\
1 & 2
\end{array}\right] \quad R=R_{1}+R_{2}=\left[\begin{array}{ll}
1 & 1 \\
1 & 2
\end{array}\right]
$$

and finally

$$
G=G_{1}+G_{2}=-P^{-1} B^{T} R=\left[\begin{array}{ll}
-1 & -2
\end{array}\right] \quad A+B G=\left[\begin{array}{rr}
0 & 1 \\
-1 & -2
\end{array}\right]
$$

We will now solve the same problem by using Algorithm 5.1 directly. We get

$$
\left[\begin{array}{ll}
1 & 0 \\
0 & 1
\end{array}\right]\left[\begin{array}{l}
q_{11} \\
q_{22}
\end{array}\right]=\left[\begin{array}{l}
1 \\
2
\end{array}\right]=\left[\begin{array}{l}
p_{2}-0 \cdot q_{12}-0 \\
p_{1}-0 \cdot q_{12}-0
\end{array}\right]=\left[\begin{array}{l}
p_{2} \\
p_{1}
\end{array}\right]
$$

From this we get the general solution

$$
Q=\left[\begin{array}{ll}
1 & c \\
c & 2
\end{array}\right], \quad c \in \mathbf{R}^{1}
$$

We see that there are an infinite number of solutions, but $R=R^{T}>0$ is only guaranteed when $Q>0$. This result was not found from the first procedure.

\subsection{Example 4}

Consider the system

$$
A=\left[\begin{array}{rr}
-6 & 5 \\
5 & -6
\end{array}\right] \quad H=\left[\begin{array}{ll}
1 & 0 \\
0 & 1
\end{array}\right]
$$

The system eigenvalues are

$$
\lambda_{1}(A)=-11, \quad \lambda_{2}(A)=-1
$$

The eigenvalues of the optimal closed loop system are specified as

$$
s_{1}(A+B G)=-14, \quad s_{2}(A+B G)=-14
$$

We use Algorithm 5.1 directly and find that $q_{12}$ must satisfy the inequality

$$
J_{I}\left(q_{12}\right)=-4 q_{12}^{2}+480 q_{12}+14400 \geqslant 0
$$

The inequality is satisfied only for $q_{12}=60$. For this value we get the only solution

$$
Q=\left[\begin{array}{cc}
135 & 60 \\
60 & 135
\end{array}\right]
$$




\section{Concluding remarks}

1. We have discussed a new method for solving the linear quadratic pole placement problem in detail. The method is numerically stable and has considerable advantages over the traditional diagonalization approach. Two eigenvalues can be moved to new real or complex locations in each step, and only a Riccati equation of an order of not more than two has to be solved. The final solution to the Riccati equation for the $n$-dimensional problem is guaranteed to be symmetrical if the two dimensional Riccati equation is solved symmetrically. Note that a symmetrical solution is not guaranteed when using Laub's (1979) method.

2. The new method gives the answer to the question about whether it is possible to determine a state weight matrix $Q$ with a given control weight matrix $P$ so that the closed loop system obtains a set of prescribed eigenvalues. This method does not only provide a yes/no answer but also gives us all such matrices (for a second order system). It could be an infinite number, a constrained space or none.

3. For a second order (sub) system we have presented the general relations between the state weight matrices $Q$ and the closed loop eigenvalues. From this result we have been able to derive the maximum possible imaginary part of the eigenvalues in a $L Q$-optimal system, irrespective of how the state weight matrix $Q$ is chosen.

4. This method can be used to determine all state weight matrices $Q$ (if there are any) that correspond to a set of prescribed eigenvalues (for a second order system). It is known that the behaviour of a system under feedback not only is dependent on the eigenvalues but also on the eigenvectors. As a result of this method we have the freedom to choose the corresponding eigenvectors such that some performance criteria are optimized.

5. This method may be used in the same way to design optimal estimators with prescribed eigenvalues. The method can also be used on discrete systems.

6. The implementation of the algorithm is simple. We recommend the use of sequence ORTHES and ORTRAN to reduce a general matrix by orthogonal similarity transformations to upper Hessenberg form. These routines are all available in EISPACK (Smith 1976). The reduction to real Schur form is done by Stewart's (1976) HQR3 software. The reordering of block $T_{j j}$ (corresponding to the eigenvalues which are to be moved) to position $T_{22}$ is done by the application of the HQR3 routine EXCHNG.

\section{REFERENCES}

Amin, M. A. (1985). Optimal pole shifting for continuous multivariable systems. Int. J. Control, 41, 701-707.

Amin, M. A., and HASSAN, M. M. (1985). Comments on 'On optimal pole assignment in a specified region'. Int. J. Control, 41, 1631-1632.

ANDERSON, B. D. O., and MOORE, J. B. (1969). Linear system optimisation with prescribed degree of stability. I.E.E.E. Trans. on Automatic Control, 116, 2083-2087.

Bar-Ness, Y. (1978). Optimal closed-loop poles assignment. Int. J. Control, 27, 421-430.

DICKMAN, A. (1987). On the robustness of multivariable linear feedback systems in state-space representation. I.E.E.E. Trans. on Automatic Control, 32, 407-410.

Doyle, J. C., Wall, J. E., and Stein, G. (1982). Performance and robustness analysis for structured uncertainty. Proceedings 21st I.E.E.E. Conf. Decision and Control, Orlando, Florida (I.E.E.E., New York), pp. 629-636. 
Gulub, G. H., and Van LoAn, C. F. (1983). Matrix Computations (North Oxford Academic Publishers Ltd, England) ISBN 0-946536-05-8.

GRAUPE, D. (1972). Derivation of weighting matrices towards satisfying eigenvalue requirements. Int. J. Control, 16, 881-888.

HEGER, F., and FRANK, P. M. (1984). Linear regulators with prescribed eigenvalues for a family of linear systems. In S. G. Tzatestas (ed.), Multivariable Control (D. Reidel Publishing Company), pp. 281-292.

JUANG, J. C., and LEE, T. T. (1984). On optimal pole assignment in a specified region. Int. J. Control, 40, 65-79.

Kalman, R. E. (1964). When is a linear system optimal? Trans. A.S.E.M. 86 D, pp. 51-60.

KAWASAKI, M., and SHIMEMURA, E. (1983). Determining quadratic weighting matrices to locate poles in a Specified region. Automatica, 19, 557-560.

KaWASAKI, M., and ShimemURA, E. (1988). Pole placement in a specified region based on a linear quadratic regulator. Int. J. Control, 48, 225-240.

LAUB, A. J. (1979). A Schur method for solving algebraic Riccati equations. IEEE Trans. on Automatic Control, 24, 913-921.

Medanic, J., TharP, H. S., and PerkinS, W. R. (1988). Pole placement by performance criterion modification. IEEE Trans. on Automatic Control, 33, 469-472.

Moliniari, B. P. (1977). The time-invariant linear-quadratic optimal control problem. Automatica, 13, 347-357.

Sмгтн, B. T. et al.(1976). Matrix Eigensystems Routines - Eispack Guide, 2nd ed. (Lecture notes in Computer Science), vol. 6 (Springer-Verlag, New York).

SAIF, M. (1989). Optimal linear regulator pole-placement by weight selection. Int. J. Control, 50, 399-414.

SHIEH, L. S., DIB, H. M. and MCINNIS, B. C. (1986). Linear quadratic regulators with eigenvalue placement in a vertical strip. IEEE Trans. on Automatic Control, 31, 241-243.

SHIEH, L. S., DiB, H. M., and GANESAN, B. (1988). Linear quadratic regulators with eigenvalue placement in a specified region. Automatica, 24, 819-823.

SolHEIM, A. O. (1972). Design of optimal control systems with prescribed eigenvalues. Int. $J$. Control, 15, 143-160.

STEWART, G. W. (1976). Algorithm 506 HQR3 and EXCHNG: Fortran subroutines for calculating and ordering the eigenvalues of a Real Upper Hessenberg Matrix. ACM Trans. Math. Soft., 2, 275-280.

Tharp, H. S. (1989). Optimal pole-placement in discrete systems. Proceedings of the American Control Conference, Pittsburgh, Pennsylvania, pp. 1286-1291, published by IEEE Service Center.

VARGA, A. (1981). A Schur method for pole assignment. IEEE Trans. on Automatic Control, 26, 517-519.

\section{Appendix}

The coefficients in equations (22) and (23) are given below

$$
\begin{aligned}
& a_{0}=h_{11} h_{22}-h_{12}^{2}=\operatorname{det} H \\
& a_{1}=h_{11} t_{22}^{2}-2 h_{12} t_{12} t_{22}+h_{22} t_{12}^{2} \\
& a_{2}=h_{11} t_{21}^{2}-2 h_{12} t_{11} t_{21}+h_{22} t_{11}^{2} \\
& b_{1}=h_{11} \\
& b_{2}=h_{22} \\
& d_{1}=2 h_{12} \\
& d_{2}=t_{11}^{2}+t_{22}^{2}+2 t_{12} t_{21}=\operatorname{tr}\left(T^{2}\right) \\
& k_{1}=2\left(h_{12} t_{21}-h_{22} t_{11}\right) t_{12}+2\left(-h_{11} t_{21}+h_{12} t_{11}\right) t_{22} \\
& k_{2}=\left(t_{11} t_{22}-t_{12} t_{21}\right)^{2}=(\operatorname{det} T)^{2}
\end{aligned}
$$


The coefficients in the inequality (35) that constrains $q_{12}$ is given below. Only $c_{2}$ and $c_{3}$ are dependent on the location of the new eigenvalues

$$
\begin{aligned}
& c_{1}=-4 a_{0}^{3}=-4(\operatorname{det} H)^{3} \\
& c_{2}=g_{1} p_{1}+g_{2} \\
& c_{3}=f_{1} p_{1}^{2}+f_{2} p_{1}+f_{3}+f_{4} p_{2}
\end{aligned}
$$

where the coefficients $g_{i}$ and $f_{i}$ are only dependent on the elements in matrices $T$ and $H$.

$$
\begin{aligned}
& g_{1}=-2 a_{0}^{2} d_{1} \\
& g_{2}=a_{0}\left(4 b_{1} b_{2} k_{1}+2 d_{1}\left(a_{0} d_{2}-a_{1} b_{2}-a_{2} b_{1}\right)\right) \\
& f_{1}=a_{0}^{2} \\
& f_{2}=-2 a_{0}\left(a_{0} d_{2}-a_{1} b_{2}-a_{2} b_{1}\right) \\
& f_{3}=\left(a_{0} d_{2}-a_{1} b_{2}-a_{2} b_{1}\right)^{2}+4 b_{1} b_{2}\left(a_{0} k_{2}-a_{1} a_{2}\right) \\
& f_{4}=-4 a_{0} b_{1} b_{2}
\end{aligned}
$$

The coefficients in equations (40) and (41) are given by

$$
\begin{aligned}
& v_{1}=\frac{-b_{2} k_{1}+a_{2} d_{1}}{a_{1} b_{2}-a_{2} b_{1}} \\
& v_{2}=\frac{b_{2}\left(p_{2}-k_{2}\right)-a_{2}\left(p_{1}-d_{2}\right)}{a_{1} b_{2}-a_{2} b_{1}} \\
& w_{1}=\frac{b_{1} k_{1}-a_{1} d_{1}}{a_{1} b_{2}-a_{2} b_{1}} \\
& w_{2}=\frac{-b_{1}\left(p_{2}-k_{2}\right)+a_{1}\left(p_{1}-d_{2}\right)}{a_{1} b_{2}-a_{2} b_{1}}
\end{aligned}
$$

The Schur formula is given by

$$
\operatorname{det}\left[\begin{array}{ll}
A & D \\
C & B
\end{array}\right]=\operatorname{det} A \operatorname{det}\left[B-C A^{-1} D\right]
$$

